\title{
Chaotic Compressed Sensing and Its Application to Magnetic Resonance Imaging
}

\author{
Nguyen Linh-Trung ${ }^{1}$, Truong Minh-Chinh ${ }^{1}$, Tan Tran-Duc ${ }^{1}, \mathrm{Ha} \mathrm{Vu}_{\mathrm{Le}}{ }^{1}, \mathrm{Minh}_{\mathrm{Ngoc}} \mathrm{Do}^{2}$ \\ ${ }^{1}$ Faculty of Electronics and Telecommunications, University of Engineering and Technology, Vietnam \\ National University Hanoi, 144 Xuan Thuy, Cau Giay, Hanoi, Vietnam \\ 2 Department of Electrical \& Computer Engineering, University of Illinois at Urbana-Champaign, \\ Illinois, USA
}

Correspondence: Nguyen Linh-Trung, linhtrung@vnu.edu.vn

Manuscript communication: received 10 October 2013, accepted 20 March 2014

\begin{abstract}
Fast image acquisition in magnetic resonance imaging (MRI) is important, due to the need to find ways that help relieve patient's stress during MRI scans. Methods for fast MRI have been proposed, most notably among them are pMRI (parallel MRI), SWIFT (SWeep Imaging with Fourier Transformation), and compressed sensing (CS) based MRI. Although it promises to significantly reduce acquisition time, applying CS to MRI leads to difficulties with hardware design because of the randomness nature of the measurement matrix used by the conventional CS methods. In this paper, we propose a novel method that combines the above-mentioned three approaches for fast MRI by designing a compound measurement matrix from a series of single measurement matrices corresponding to pMRI, SWIFT, and CS. In our method, the CS measurement matrix is designed to be deterministic via chaotic systems. This chaotic compressed sensing (CCS) measurement matrix, while retaining most features of the random CS matrix, is simpler to realize in hardware. Several compound measurement matrices have been constructed and examined in this work, including CCS-MRI, CCS-pMRI, CCS-SWIFT, and CCS-pSWIFT. Simulation results showed that the proposed method allows an increase in the speed of the MRI acquisition process while not compromising the quality of the acquired MR images.
\end{abstract}

Keywords- magnetic resonance imaging (MRI), SWeep Imaging with Fourier Transformation (SWIFT), parallel MRI, fast acquisition, compressed sensing, measurement matrix, chaotic filter, deterministic design.

The work was supported by Project 57/2011/HDDT from the Asia Research Center of Vietnam National University Hanoi.

\section{INTRODUCTION}

The concept of information was proposed by Shannon in 1948 [1] where the information of a source is statistically defined via entropy. Traditionally, the acquisition (also called, sampling or sensing) of the data from a source follows the Nyquist law where the source signal is sampled at a frequency larger than or equal to twice the maximum frequency present in the frequency content of the signal, without much concerning the information of the source. Digital information processing is then applied in order to extract the information from the digitally acquired data. Therefore, the acquisition is to sense the data of the source.

Recently, new perspectives have been proposed that shift the concern of many researchers from data to more on information. One of such perspectives is a new sensing modality called compressive sensing (CS) $[2,3]$. Instead of sensing the data of the source, CS tries to sense the information directly from the source. By doing so, the data acquired as the result of the CS process would be different from the data acquired by the traditional sensing processes. Taking an example of sensing a voice, Nyquist sampling will give us data in the time domain (also called the ambient domain which is well understood). However, with CS, different data are obtained and they are represented in another domain that depends on the acquired method. This domain is said to be holographic because it is not presented in the normal way of understanding.

Assume that the signal of interest is sparse (or compressible) in some specific domain. For example, a real communication channel would induce errors on a transmitted signal. Given that the transmitted signal is channel-coded, the resulting received signal vector is the sum of the transmitted codeword vector and an error vector. This error vector is sparse, meaning that there are only a few nonzero entries. Another example is that a natural image is compressible in the wavelet domain; only a few wavelet coefficients are significant. Hereafter, we will only focus on the sparsity of the signal of interest. This sparsity represents the information of the signal. CS then aims to sense this information (not the data) directly from the signal, by projecting the signal on to a random linear vector space; the sensing system can be modeled as a matrix and this measurement matrix is random. Because of the sparsity, CS acquires far less samples when compared to Nyquist sampling. The signals are then reconstructed/recovered using a sparse approximation technique, such as $\ell_{1}$ 
optimization (e.g., Basis Pursuit) or greedy algorithms (e.g., Orthogonal Matching Pursuit). Exact construction of the signal in CS is guaranteed if the sparsity domain is incoherent to the sensing domain [4]. However, designing the sensing system to represent a random measurement matrix is difficult in practice. A deterministic CS method was introduced by Linh-Trung et al. in [5], called chaos filter, exploiting chaotic systems for CS.

Magnetic resonance imaging (MRI), based on Fourier transform, is an imaging technique to visualize the internal structures of the object in detail [6]. More efficient acquisition in MRI, especially fast acquisition, is of interest to many researchers. It is important to reduce the scanning time on patients in order to avoid physiological effects, to overcome physical constraints of the imaging system, or to meet timing requirements when imaging dynamic structures or processes. Traditionally, parallel imaging is designed to speed up the image in MRI. In parallel imaging, a reduced data set in the phase encoding directions of $k$-space is acquired to reduce acquisition time, combining the signal of $L$ coils [7]. These coils operate simultaneously, giving a set of MR signals with different information at once. Each coil acquires data corresponding to a portion of the imaging object. By combining the data obtained from the separate coils, the full MRI image can be reconstructed.

While many works on the theory of CS (whether being random or deterministic) were being developed, Lustig has successfully shown that random CS can be applied to speed up the image acquisition in Magnetic Resonance Imaging (MRI) [8]; the method is called sparse-MRI. The sparsity of the MRI images in the wavelet domain is exploited to perform CS. Recently, Puy et al. has applied CS to MRI with a linear frequency-modulated excitation [9]. Inspired by the sparse-MRI method for fast image acquisition, in this paper we study chaotic CS for MRI.

We first give a deterministic perspective on CS. In this context, firstly, we design the entries of the measurement matrix as values generated from a deterministic chaotic system. Secondly, we use some families of deterministic signals to design the measurement matrix. Lastly, we generalize the deterministic structure of the measurement matrix by decomposing into a number of deterministic matrices that capture different structures in the sensing system.

Next, we illustrate the view of chaotic CS on MRI. The reason is that with MRI we can implement the decomposition of the measurement matrix under different specific MRI techniques. They could be a Fourier matrix (with standard MRI), undersampled permutation matrix (with sparse-MRI), hyperbolic-secant matrix (with SWeep Imaging with Fourier Transformation (SWIFT) [10]), etc.

The paper is organized as follows. Section 2 first describes the principle of compressed sensing, and then presents the fundamental of chaotic compressed sensing. Section 3 explains the principles of twodimensional (2D) MRI acquisition and the specialized methods for fast MRI acquisition using SWIFT and parallel MRI, all in view of algebraic formulation. Section 4 describes the first method that combines Fourier imaging and chaotic CS (CCS-MRI). Section 5 describes the second method, CCS-SWIFT, which integrates CCSMRI with SWIFT to exploit a frequency-swept excitation pulse and virtually simultaneous signal acquisition in a time-shared mode. These two methods have been given in our previous papers and are presented here for the next method which clearly shows the view of the compound measurement matrix. Section 6 describe the third method, CCS-pSWIFT, which further applies parallel imaging to CCS-SWIFT. Simulation results to illustrate the effectiveness of each method in terms of normalized image reconstruction error are also given accordingly. Finally, Section 7 concludes the paper and gives discussions on the proposed methods.

\section{Chaotic Compressed Sensing}

\subsection{Fundamentals of Compressed Sensing}

We briefly presents the fundamental of CS in this section, restricting our attention to the discrete-to-discrete formulation of CS for simplicity.

Let $\mathbf{x} \in \mathbb{R}^{N}$ be the unknown signal of interest, to be sensed by CS. Suppose that $\mathbf{x}$ is $K$-sparse, that is $\mathbf{x}$ admits a sparse representation $\mathbf{x}=\mathbf{\Psi} \boldsymbol{\alpha}$ by the representation basis $\boldsymbol{\Psi}=\left[\psi_{1}, \ldots, \psi_{N}\right]$ and the coefficient vector $\alpha \in \mathbb{R}^{N}$ has $K$ nonzero values out of $N$ values. Thus, $\Psi$ is also called the sparsifying matrix.

A linear system, represented by a random measurement matrix $\boldsymbol{\Phi} \in \mathbb{R}^{M \times N}$, is designed to sense $\mathbf{x}$ by the following model:

$$
\mathbf{y}=\boldsymbol{\Phi} \mathbf{x}
$$

where $\mathbf{y} \in \mathbb{R}^{M}$ is the measurement vector. This linear system is underdetermined; that is, $M<N$.

From only a small number of $M$ measurements and a priori information about $\boldsymbol{\Psi}$ and $\boldsymbol{\Phi}, \mathrm{CS}$ aims to determine/recover/reconstruct $\mathbf{x}$. Since the system is underdetermined, it is necessary to rely on additional constraints in order to solve (1). One important constraint is the sparsity of $\mathbf{x}$ that we have assumed. Another constraint is the so-called Restricted Isometry Property (RIP) which is explained as follows. Let $\boldsymbol{\Theta}=\boldsymbol{\Phi} \boldsymbol{\Psi}$, then the model (1) is rewritten as $\mathbf{y}=\boldsymbol{\Theta} \boldsymbol{\alpha}$. Under the RIP, $\Theta$ approximately preserves the length of $K$-sparse signals; that is, all subsets of $K$ columns of $\Theta$ are nearly orthogonal. In other words, $\Theta$ is an almost orthonormal system when restricted to sparse linear combinations.

One way to satisfy the RIP is to satisfy the incoherence condition. The coherence, denoted as $\mu(\boldsymbol{\Phi}, \boldsymbol{\Psi})$, measures the largest correlation between any two columns of $\boldsymbol{\Phi}$ and $\boldsymbol{\Psi}$ and is constrained in the range $[1, \sqrt{N}]$. The incoherence corresponds to $\mu=1$ and compressed sensing takes place when $\mu$ is close to 1 . Note that, while the sparsity is related to the signal of interest, the incoherence is related to the sensing modality.

When the random entries of $\boldsymbol{\Phi}$ are independently and identically Gaussian distributed with zero mean and variance of $1 / M$ (hence, $\mu$ is small), if $M \geq$ 
$C K \log (N / K)$ for some positive constant $C$, then exact reconstruction of $\alpha$ (or essentially $\mathbf{x}$ because $\Psi$ is known) can be achieved with an overwhelming probability by solving the following $\ell_{1}$-minimization problem:

$$
\boldsymbol{\alpha}=\arg \min _{\boldsymbol{\alpha}^{\prime}}\left\|\boldsymbol{\alpha}^{\prime}\right\|_{1}, \quad \text { subject to } \boldsymbol{\Theta} \boldsymbol{\alpha}^{\prime}=\mathbf{y},
$$

where $\|\cdot\|_{1}$ denotes the norm- 1 operator. If there exists an additive noise in the measurements, the optimization problem is reformulated as follows:

$$
\boldsymbol{\alpha}=\arg \min _{\boldsymbol{\alpha}^{\prime}}\left\|\boldsymbol{\alpha}^{\prime}\right\|_{1}, \quad \text { subject to }\left\|\boldsymbol{\Theta} \boldsymbol{\alpha}^{\prime}-\mathbf{y}\right\|_{2}<\epsilon,
$$

where $\epsilon$ is a threshold that controls the fidelity of the reconstruction $\Theta \alpha^{\prime}$ with respect to the measure data $\mathbf{y}$, and is normally set below the noise level.

\subsection{Compressed Sensing using Chaos Filters}

Conventionally, the measurement matrix $\boldsymbol{\Phi}$ is random and the signal reconstruction algorithm reconstructs the signal $\mathbf{x}$, or, equivalently, its $K$-sparse coefficient vector $\alpha$ from $M$ random measurements in the vector $\mathbf{y}$.

It is known that the hardware implementation of a deterministic system is often simpler than that of a random one. Therefore, we makes $\boldsymbol{\Phi}$ chaotic, which is generated from a deterministic system. Inspired by the design of the random filter for CS in [11], a design of a chaotic filter for CS was proposed in [12], where a chaotic $\boldsymbol{\Phi}$ is constructed from a chaotic sequence $h_{G}(n)$. This sequence is obtained by first generating the Logistic map

$$
h(n+1)=\rho h(n)(1-h(n)),
$$

and then converting it by the Logit Transform to be Gaussian-like as

$$
h_{G}(n)=\ln \left[\frac{h(n)}{1-h(n)}\right] .
$$

For $h(n)$ to be chaotic, the control parameter $\rho$ must be equal to 4 . The initial condition $h(0)$ is very sensitive in the sense that the output chaotic sequence is completely different for a small change of $h(0)$. More details on how to construct $\boldsymbol{\Phi}$ from $h_{G}(n)$ can be found in [12]. After that, the reconstruction is performed using the Orthogonal Matching Pursuit technique.

Figure 1 shows the performance of both random and chaos filters of length $L$ in reconstructing the original signal $\mathbf{x}$, in terms of the probability of exact reconstruction. The signal of interest is a sequence of length 512 and is sparse in the time domain with $K=20$ spikes; the locations of the spikes were randomly generated. The coefficients of the random filter were obtained from the Gaussian distribution with zero mean and unit variance. The coefficients of the chaotic filter were generated by the Gaussian-Logistic map as explained above. It can be seen that the chaotic filter offers a performance similar to that of the random filter, except when the filter length is very short $(L=32)$.

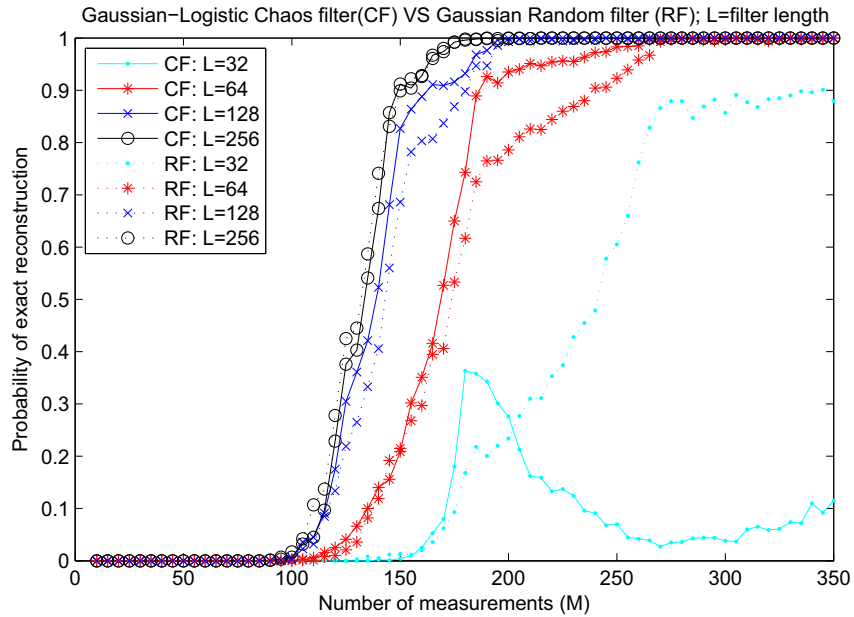

Figure 1. Performance comparison of Gaussian-Logistic chaos and Gaussian random filters.

\section{Magnetic Resonance Imaging}

\subsection{Standard 2D Image Acquisition in Magnetic Resonance Imaging}

Magnetic resonance imaging (MRI) is a valuable diagnostic research that has been used for medical analysis since the 1980s. MRI is a noninvasive and nonradioactive technology. An MRI test is often used as a part of a medical examination because MRI could view an object by two-dimensional (2D) or three-dimensional (3D) images. MRI is based on the phenomenon of magnetic resonance of tissue nuclei present in the object under imaging. It produces images of the human body with a quality higher than X-ray images.

During an MRI scan, some radio-frequency (RF) waves are released in pulses through the object, exciting movement in hydrogen nuclei in the object. When the radio waves stop, the hydrogen nuclei relax and release energy. Different parts of the object have different characteristic patterns of how they are affected by the RF waves. The resonance information of the nuclei can be picked up by an RF receiving coil upon the stop of the excitation RF waves.

A trained radiologist reads these images presented in sections or slices. We present here the simplest case of acquisition of a full 2D digital image of an object, for example a brain slice as shown in Figure 3(a), to explain how the image acquisition is done. During a series of RF excitations each of which encodes the 2D location information of a particular point on the brain slice, the receiving coil detects an analog MRI time signal which contains the resonance information at all encoded locations. The encoded locations are represented in a temporary image space, which is called $k$-space. The changes of locations in the $k$-space during the acquisition time often form a smooth trajectory, such as shown in Figure 2(a). Most of the encoded information concentrates around the origin of the $k$ space, and the density of the $k$-space approximately follows a power decay law. A digital MRI signal is then obtained by sampling the time $(t)$ and the $k$-space. 


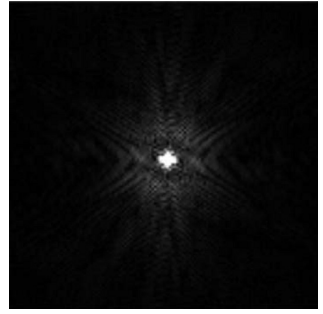

(a)

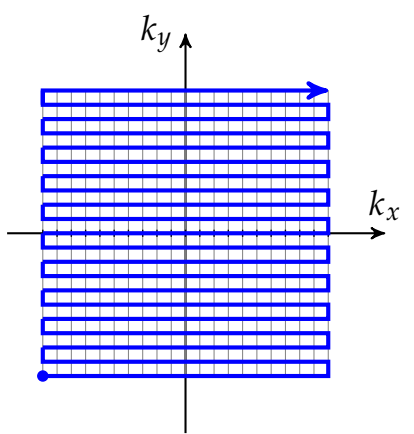

(b)

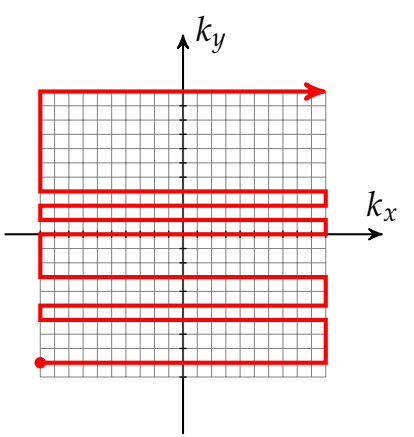

(c)
Figure 2. $k$-space of a brain MR image. (a)- analog acquisition, (b)linear sampling of (a), (c)- linear undersampling of (a). In (c), a binary mask (of $128 \times 128$ points) is applied to (a).

Next, the digital MRI image of the brain slice can be reconstructed by applying a reconstruction algorithm on the digital MRI signal. The image reconstruction can be done in either the image domain or the $k$-space domain. For example, the digital MRI image can be obtained by a 2D Fourier transform on the digital MRI signal from the $k$-space to the pixel domain.

Let $m(x, y)$ be the image of the object that need to be reconstructed. The digital MRI signal acquired by the receiving coil is given by the following imaging equation:

$$
v\left(k_{x}, k_{y}\right)=\sum_{n_{x}=0}^{N_{x}-1} \sum_{n_{y}=0}^{N_{y}-1} m\left(n_{x}, n_{y}\right) e^{-j\left(k_{x} x+k_{y} y\right)},
$$

where $k_{x}$ and $k_{y}$ respectively encode the $k$-space information of locations corresponding to the $x$ and $y$ directions of the image, $N_{x}$ and $N_{y}$ respectively are the numbers of pixels along the $x$ and $y$ axes of the image. The $k$-space contains points $k=\left\{k_{x}, k_{y}\right\}$. Note that, the discrete representation in (6) corresponds to a linear full-sampling in the $k$-space along a Cartesian trajectory as shown in Figure 2(b). In matrix form for the imaging equation (6), the MRI signal acquired in the $k$-space $v$ is expressed as

$$
v=\mathbf{F m}+\mathbf{n},
$$

where $\mathbf{m}$ is the image vector of length $N$ to be acquired, F is the Fourier matrix of size $N \times N$, and $\mathbf{n}$ is the additive noise.

Then, $\mathbf{m}$ can be reconstructed by solving the following least-norm problem:

$$
\arg \min \|\mathbf{m}\|, \quad \text { subject to }\|\boldsymbol{v}=\mathbf{F m}\| .
$$

to obtain the unique solution

$$
\hat{\mathbf{m}}=\mathbf{F}^{*}\left(\mathbf{F F}^{*}\right)^{-1} \boldsymbol{v}
$$

where the operator $(\cdot)^{*}$ denotes the conjugate transpose.

\subsection{Specialized MRI as SWeep Imaging with Fourier Transform (SWIFT)}

The MRI technology has continued to develop and is now an effective diagnostic tool in clinical medicine. However, conventional MRI methods could not evaluate hard tissues in the body due to its inability to capture the rapidly decaying signals of these tissues. In this case, the echo time, which is the duration of the RF excitation and signal acquisition process, is very small. In 2006, Idiyatullin et al. proposed a new method called SWeep Imaging with Fourier Transform (SWIFT) in order to overcome this advantage of conventional MRI [10]. The breakthrough idea of SWIFT is the use of the time-shared RF excitation and acquisition to capture signals originating from molecules which have very short transverse relaxation times. This RF excitation pulse is generally expressed by

$$
g(t)=\omega(t) \exp \left\{-j \int_{0}^{t}\left(\omega_{\mathrm{RF}}(\tau)-\omega_{c}\right) d \tau\right\}
$$

where the amplitude modulation, $\omega(t)$, and the frequency modulation, $\omega_{\mathrm{RF}}(t)$. These modulating signals are designed based on the family of adiabatic hyperbolic-secant $\left(\mathrm{HS}_{n}\right)$ pulses

$$
f_{n}(t)=\operatorname{sech}\left[\beta\left(\frac{2 t}{T_{p}}-1\right)^{n}\right]
$$

as follows:

$$
\begin{aligned}
\omega(t) & =\gamma B_{1 \max } f_{n}(t) \\
\omega_{\mathrm{RF}}(t) & =\omega_{c}+2 A\left(\frac{\int_{0}^{t} f_{n}^{2}(\tau) d \tau}{\int_{0}^{T_{p}} f_{n}^{2}(\tau) d \tau}-\frac{1}{2}\right) .
\end{aligned}
$$

Above, $n$ is a shape vector (typically, $n \geq 1$ ), $\beta$ is a truncation factor (usually, $\beta \approx 5.3$ ), $T_{p}$ is the pulse length, $\gamma$ is the gyromagnetic ratio, $B_{1 \max }$ is the maximum amplitude of the RF pulse, $\omega_{c}$ is the center angular frequency, and $A$ is the bandwidth of the pulse $\left(-A \leq \omega_{\mathrm{RF}}-\omega_{c} \leq A\right)$. In SWIFT, during the excitation of the HSn pulse from 0 to $T_{p}$ seconds, the transmitter is repeatedly turned on and off to enable the acquisition in short intervals of time, thus the acquisition is virtually performed simultaneously with the excitation [10] [13].

In comparison with the standard MRI acquisition, the excitation in MRI acquisition is an impulse function while that in SWIFT is the hyperbolic-secant signal $g(t)$. In other words, the image is viewed as being premodulated by $g(t)$. Then, the imaging equation in (6) becomes

$$
v\left(k_{x}, k_{y}\right)=\sum_{n_{x}=0}^{N_{x}-1} \sum_{n_{y}=0}^{N_{y}-1} m\left(n_{x}, n_{y}\right) g\left(n_{x}, n_{y}\right) e^{-j\left(k_{x} x+k_{y} y\right)},
$$


and can be expressed, with additive noise, in matrix form as

$$
v=\mathbf{F G m}+\mathbf{n},
$$

where $\mathbf{G}$ is a diagonal matrix of size $N \times N$ whose diagonal elements are obtained from the hyperbolicsecant signal $g(t)$ [14].

Consequently, the image $\mathbf{m}$ is the unique solution to the following least-norm problem:

$$
\hat{\mathbf{m}}=\arg \min \|\mathbf{m}\|, \quad \text { subject to }\|\boldsymbol{v}=\mathbf{F G m}\| \text {. }
$$

\section{Chaotic Compressed Sensing for MRI (CCS-MRI)}

In MRI, the contrast information is concentrated at low spatial frequencies; that is, (near the center) of the $k$ space. MRI images are sparse in the wavelet domain. Lustig et al. has successfully designed a random CS matrix that can be applied to remove the residue information and hence speed up the image acquisition in MRI and called the method sparse-MRI [8]. However, designing a random system is difficult in practice. Thus, we applied Gaussian-Logistic chaos to create a randomlike sampling matrix $\mathbf{C}$ instead of full-sampling as shown in the imaging equation of (7). By using this under-sampling technique, (7) can be rewritten as

$$
v=\mathbf{C F m}+\mathbf{n} \text {. }
$$

The image is reconstructed by solving the following optimization [8]:

$$
\hat{\mathbf{m}}=\arg \min _{\mathbf{m}}\left\{\|\mathbf{C F m}-\boldsymbol{v}\|_{2}^{2}+\lambda\|\mathbf{\Psi} \mathbf{m}\|_{1}\right\},
$$

using the non-linear conjugate gradient (NCG) algorithm. In (18), $\lambda$ is a regularization parameter and $\boldsymbol{\Psi}$ is the sparsifying matrix in the wavelet domain. We can see that, for CCS-MRI, the measurement matrix is given by $\boldsymbol{\Phi}=\mathbf{C F}$ of size $M \times N$.

We performed simulation to confirm the ability of CCS-MRI [15]. In this simulation, the data source in use is a brain slice of $128 \times 128$ pixels, as shown in Figure 3(a). The Logistic map in (4) was simulated with the control parameter $\rho=4$ and the initial condition $h(0)=3$.

Figure 3(b) shows the aliasing image after reconstruction when the $k$-space was under-sampled linearly at the compression ratio of $r=0.3$, where this ratio is defined as $r=M / N$. Figure 3(c) shows the reconstructed image obtained by the CCS-MRI method, also at $r=0.3$. The aliasing effect in the reconstructed image was reduced.

To compare the efficiency of the CCS-MRI method with that of the sparse-MRI (random CS-MRI) method, we acquired brain MRI data for a series of compression ratios $r$ from 0.1 to 0.5 . Then, we determine for each compression ratio the normalized error in the reconstructed image $\hat{\mathbf{m}}$ with respect to the original image $\mathbf{m}$ as

$$
e=\frac{1}{N_{x} \times N_{y}} \frac{\sum_{i=1}^{N_{x}} \sum_{j=1}^{N_{y}}\left|\mathbf{m}_{i j}-\hat{\mathbf{m}}_{i j}\right|}{M},
$$

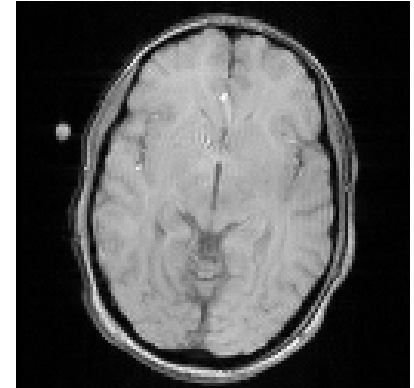

(a)

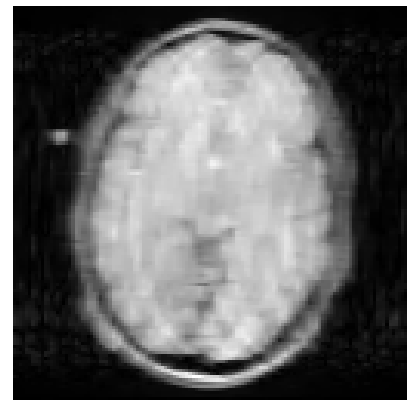

(c)

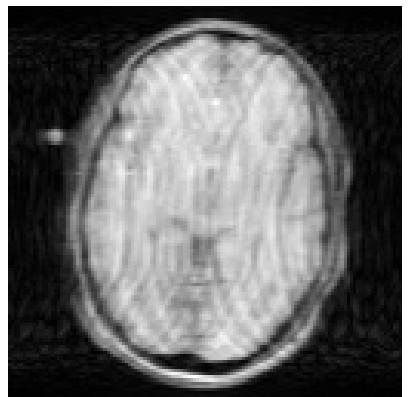

(b)

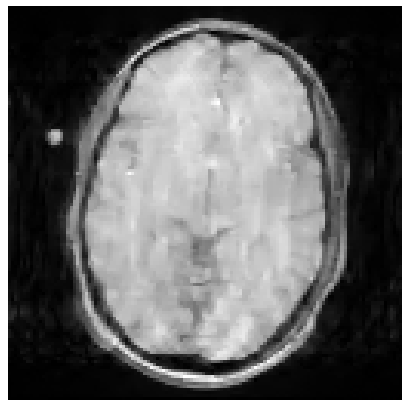

(d)
Figure 3. Original brain slice image (a), and its reconstructed images (b) without using CS, (c) with CCS-MRI and (d) with CCSSWIFT, at compression ratio $r=0.3$.

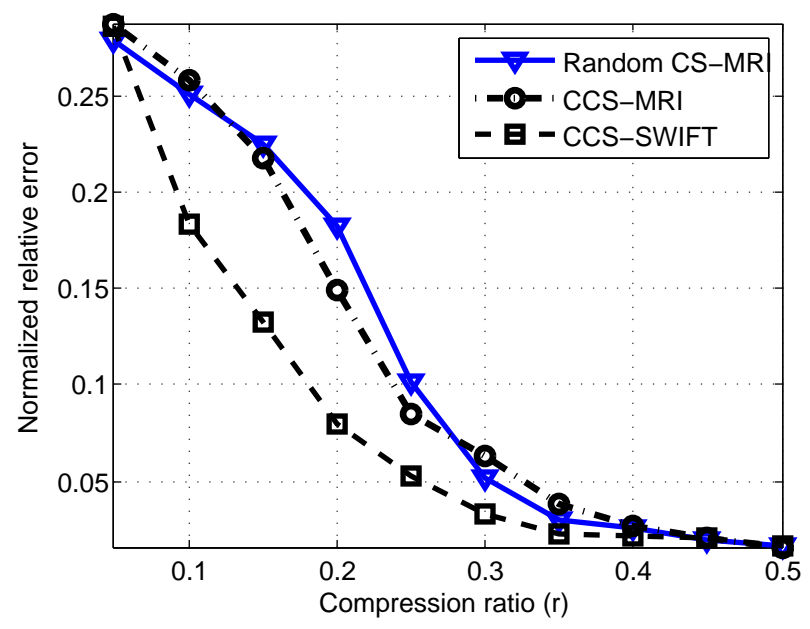

Figure 4. Normalized image relative error performances.

where $N_{x} \times N_{y}$ is the number of pixels, and $M$ is the maximum value in $\mathbf{m}$. Figure 4 shows the performance of sparse-MRI (random CS-MRI) and CCS-MRI methods. It can be seen that CCS-MRI offers a similar performance as compared to sparse-MRI.

\section{Chaotic Compressed Sensing for SWIFT (CCS-SWIFT)}

In this section, we proposed a scheme that exploits the advantages of both chaotic CS and SWIFT; the method is called CCS-SWIFT.

By using an impulse to excite a conventional MRI system, the signal output is the response of the system to the impulse, that is the MRI image. In frequency 
domain, it can be considered as pre-multiplying an identity matrix with the frequency response of the MRI system. By using hyperbolic-secant signal in the SWIFT method, the output of the SWIFT scanner in frequency domain is the multiplication of a diagonal matrix $\mathbf{G}$, which corresponds to the hyperbolic-secant signal, with the image $\mathbf{m}$, as given in (15). Consequently, based on the imaging equation of (17) for SWIFT, we have the imaging equation for the CCS-SWIFT as

$$
\begin{aligned}
v & =\mathrm{CFGm}+\mathbf{n} \\
& =\mathrm{CFm}_{1}+\mathbf{n} .
\end{aligned}
$$

We can see that, for CCS-SWIFT, the measurement matrix is now given by $\Phi=$ CFG and has a size of $M \times N$.

Note that $\mathbf{m}_{1}$ is also sparse in the wavelet domain. Therefore, the image is reconstructed by first solving the following optimization using the non-linear conjugate gradient (NCG) algorithm [8] for $\mathbf{m}_{1}$ :

$$
\hat{\mathbf{m}}_{1}=\arg \min _{\mathbf{m}_{1}}\left\{\left\|\mathbf{C F m} \mathbf{m}_{1}-\boldsymbol{v}\right\|_{2}^{2}+\lambda\left\|\mathbf{\Psi} \mathbf{m}_{1}\right\|_{1}\right\},
$$

and then solving for $\mathbf{m}$ as

$$
\hat{\mathbf{m}}=\mathbf{G}^{T}\left(\mathbf{G G}^{T}\right)^{-1} \hat{\mathbf{m}}_{1} .
$$

We analyze the CCS-SWIFT method with the same simulation scenario as described in Section 4. For the hyperbolic-secant pulse $f_{n}(t)$, we set $n=1$ [16]. Figure $3(\mathrm{~d})$ shows the reconstructed image when using the CCS-SWIFT method at a compression ratio $r=$ 0.3. Comparing that with Figure 3(c) for the CCSMRI method, it can be seen that CCS-SWIFT offers a higher quality of reconstruction than CCS-MRI. This is the result of spreading the $k$-space thanks to the broadband hyperbolic-secant signal $g(t)$, as shown in Figure 5, which helps reduces the coherence between the measurement matrix and the sparsifying matrix.

The performance of CCS-SWIFT is also shown in Figure 4 with a range of compression ratios from 0.1 to 0.5 . It can be seen that the CCS-SWIFT method outperforms both the sparse-MRI and the CCS-MRI methods, notably in the range of $[0.1,0.3]$ of the compression ratio.

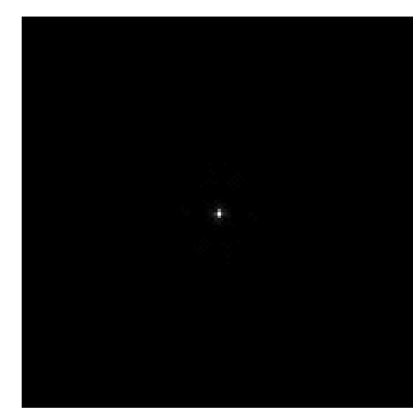

(a)

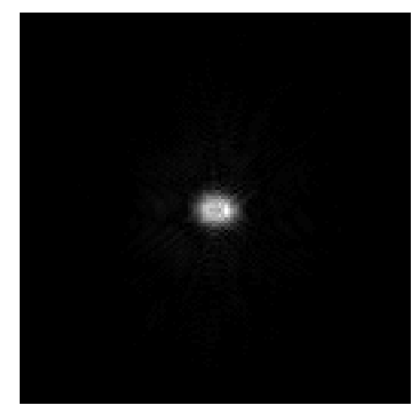

(b)
Figure 5. Effect of spreading the $k$-space in SWIFT. 5(a)- usual $k$ space with Fourier MRI, 5(b)- spreaded $k$-space with SWIFT.

\section{CCS-SWIFT with Parallel MRI (CCS-PSWIFT)}

Parallel MRI (pMRI) is known to be a method to create a special sampling matrix. When we concern to pMRI, we would like to increase the speed of the MRI acquisition by skipping a number of phase-encoding lines in the $k$ space during the MRI acquisition. The data are received simultaneously by $L$ receiver coils with distinct spatial sensitivities and are processed to reconstruct the values in the missing $k$-space lines. Thus, both random and chaotic CS can be applied to pMRI naturally [17, 18].

There are various parallel imaging methods. In this paper, we only considers a simple and effective method called Sensitivity Encoding for fast MRI (SENSE) which was proposed in [19]. Given the $k$-space is fully sampled, the imaging function of pMRI for the $i$-th coil is given as follows:

$$
\begin{aligned}
v_{i}\left(k_{x}, k_{y}\right)=\sum_{n_{x}=0}^{N_{x}-1} \sum_{n_{y}=0}^{N_{y}-1} S_{i}\left(n_{x}, n_{y}\right) \\
\times m\left(n_{x}, n_{y}\right) e^{-j 2 \pi\left(n_{x} k_{x}+n_{y} k_{y}\right)},
\end{aligned}
$$

where $S_{i}\left(n_{x}, n_{y}\right)$ is the sensitivity function of the $i$ th coil. The image acquired by each individual coil (channel) can then be expressed in matrix form as the ideal image modulated by the corresponding sensitivity function:

$$
\mathbf{m}_{i}=\mathbf{S}_{\mathbf{i}} \mathbf{m}, \quad i=1, \ldots, L .
$$

The mixed image matrix $\mathbf{m}^{*}$ of size $N L \times N$ is formed as $\mathbf{m}^{*}=\left[\mathbf{m}_{1} ; \mathbf{m}_{2} ; \ldots ; \mathbf{m}_{L}\right]$. Consequently, the image $\mathbf{m}$ can be reconstructed by

$$
\hat{\mathbf{m}}=\arg \min \|\mathbf{m}\|, \quad \text { subject to }\left\|v=\mathbf{F}_{N L} \mathbf{S m}^{*}\right\|,
$$

where $\mathbf{S}$ is a $N L \times N L$ matrix formulated from all $L$ sensitivity matrices $\mathbf{S}_{\mathbf{i}}$ and $\mathbf{F}_{N L}$ is the Fourier matrix of size $N L \times N L$ [19]. After a matrix reduction step, the imaging equation in matrix form is given by

$$
v=\mathbf{F R m}+\mathbf{n},
$$

where $\mathbf{R}$ is a reconstruction matrix of $N \times N$ derived from $\mathbf{S}$.

Now, we apply SWIFT to each coils (before applying pMRI) and then chaotic CS to construct a method of acquisition which combines chaotic CS with SWIFT and parallel MRI. By using the imaging equation of CCSSWIFT in (21), the imaging equation of the resulting CCS-pSWIFT method becomes

$$
\begin{aligned}
\boldsymbol{v} & =\mathrm{CFRGm} \\
& =\mathrm{CFRm}_{1}
\end{aligned}
$$

We can see that, for the CCS-pSWIFT method, the measurement matrix is given by $\boldsymbol{\Phi}=$ CFRG.

Therefore, the image is reconstructed by first solving the following optimization:

$$
\hat{\mathbf{m}}_{1}=\arg \min _{\mathbf{m}_{1}}\left\{\left\|\mathbf{C F R m}_{1}-\boldsymbol{v}\right\|_{2}^{2}+\lambda\left\|\mathbf{I m}_{1}\right\|_{1}\right\},
$$

and then obtain $\mathbf{m}$ as the unique solution of (23). Our scheme can be summarized in Algorithm 1. 


\section{Algorithm 1 Chaotic compressed sensing for parallel SWIFT acquisition (CCS-pSWIFT)}

Step 1: Set up for hyperbolic-secant excitation $g(t)$.

Step 2: Generate $k_{x}, k_{y}$ to be Gaussian-Logistic chaotic sequences. The number of $k_{x}, k_{y}$ based on pre-defined compression ratio $r=M / N$.

Step 3: For each channel, determine the coordinates in $k$ space based on $k_{x}, k_{y}$ and store them as a mask.

Step 4: For each channel, acquire the digital data in the $k$-space based on the mask and store them in a vector $\mathbf{y}$.

Step 5: Estimate the $L$ sensitivity maps using polynomial fitting.

Step 6: Perform SENSE reconstruction using conjugated gradient method.

Step 7: Perform SWIFT-demodulation using Equation (23).

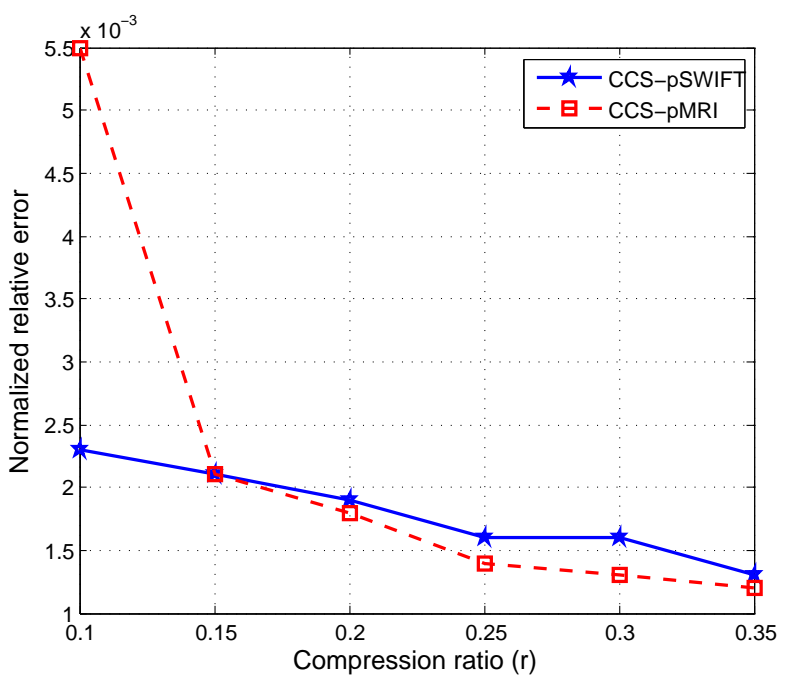

Figure 6. Performance comparison between CCS-pMRI and CCSpSWIFT.

To evaluate the CSS-pSWIFT method, the data source for simulation is the human MPRAGE data from 8channel head array coil $(L=8)$. The data was acquired with the following parameters: $\mathrm{TE}=3.45 \mathrm{~ms}$, $\mathrm{TR}=2350 \mathrm{~ms}, \mathrm{TI}=1100 \mathrm{~ms}, \mathrm{Flip}$ angle $=7 \mathrm{deg}$, slice $=1$, matrix $=128 \times 128$, slice thickness $=1.33 \mathrm{~mm}, \mathrm{FOV}=256 \mathrm{~mm}$. We acquire the data for a series of compression ratios from 0.1 to 0.35 , and then analyze the performance of both CSS-pMRI and CCSpSWIFT methods in terms of the normalized image reconstruction error using Equation (19).

As can be seen in Figure 6, it is obvious that the proposed CCS-pSWIFT method outperforms the CCSpMRI method for a range of relatively small compression ratios $(r<0.15)$. The reason is that the modulation spreads the spectrum of $\mathbf{m}$ so that the information of these vectors can be easily accessed in the $k$-space. With a higher ratio $(r>0.15)$, the role of the parallel coils is obvious when the information in the $k$-space from the multiple channels can be combined in the reconstruction process. Therefore, the proposed designs applied for parallel imaging can only show their advantage in the low range of compression ratios.

\section{Discussions}

Above, based on the CS approach, we have presented the design for a compound measurement matrix that is aimed at improving the quality of MRI images with reduced acquisition time. In the last method (CCSpSWIFT), this measurement matrix is formed by factoring up to three single measurement matrices derived respectively from the individual designs of CS, SWIFT and pMRI methods, together with the Fourier matrix which is inherent in the design of the conventional MRI method. CCS-pSWIFT is therefore capable of providing all the advantages offered by several methods in a single operation. Our designs are aimed at reducing the coherence between the compound measurement matrix and the sparsifying matrix, thanks to relying on the specialized SWIFT technique, that leads to a quality improvement for the reconstructed MRI images.

The simulations show that, with the same normalized relative error rate (e.g., $e=0.15$ ), the CCS-SWIFT method can save up to $6 \%$ of the sampling data as compared to the CCS-MRI method. Similarly, the CCSpSWIFT method can save up to $4.5 \%$ of the sampling data as compared to CCS-pMRI at the normalized relative error rate $e=2.3 \times 10^{-3}$.

Another advantage of our proposed approach in comparison with other CS-related methods is its better applicability for hardware implementation thanks to the deterministic property of the proposed measurement matrix. To facilitate practical implementations, some research efforts are focusing on designing measurement matrices that are not completely random [20] or totally deterministic [21]. In [21], several simple criteria were provided for designing several families of deterministic sensing matrices to guarantee a successful recovery of sparse signals. The criteria were also applied to random Fourier ensembles to determine bounds on the number of measurements required for sparse approximation. In general, the deterministic approach in CS provides some advantages over random CS, such as more efficient recovery time, explicit constructions, efficient storage, and tighter recovery bounds [21].

In our particular design of the measurement matrix for CS, we have chosen to use a chaotic system which is a deterministic nonlinear dynamical system that is very sensitive to initial conditions, and the time-series as the output of the chaos system is random-like. Note that, as with random CS, exact reconstruction in chaotic CS is also guaranteed [22]. The hardware implementation for a chaotic generator is just a simple circuit of a nonlinear system, and thus is simpler than that for a random generator. To generate a random sequence, one uses a hardware random number generator (HRNG) or a pseudo-random number generator (PRNG). The HRNG works based on microscopic phenomena that generate a low level, statistically random noise signal. It uses a transducer to convert some physical phenomena to an electrical signal, an amplifier to amplify this random fluctuations, and an analog-to-digital converter to convert the output into simple binary values of 0 or 1 . 
By repeatedly sampling the randomly varying signal, a sequence of random numbers is archived. The PRNG can generate random-like numbers by feedback shift registers, it is more practical than the HRNG. However, a long register is needed to generate a sequence of numbers that approximates the properties of random numbers. Therefore, a large memory and logic circuits are required.

\section{REFERENCES}

[1] C. E. Shannon, "A mathematical theory of communication," The Bell System Technical Journal, vol. 27, pp. 379$423,1948$.

[2] E. Candes, J. Romberg, and T. Tao, “Robust uncertainty principles: Exact signal reconstruction from highly incomplete frequency information," IEEE Transactions on Information Theory, vol. 52, no. 2, pp. 189-509, Feb. 2006.

[3] D. Donoho, "Compressed sensing," IEEE Transactions on Information Theory, vol. 52, no. 4, pp. 1289-1306, Apr. 2006.

[4] E. Candes and J. Romberg, "Sparsity and incoherence in compressive sampling," Inverse Problems, vol. 23, no. 3, pp. 969-985, Apr. 2007.

[5] N. Linh-Trung, D. V. Phong, Z. Hussain, H. Huynh, V. Morgan, and J. Gore, "Compressed sensing using chaos filters," in Proc. Australian Telecommunication Networks and Applications Conference (ATNAC 2008), 2008, pp. 219-223.

[6] R. R. Edelman and S. Warach, "Magnetic resonance imaging," New England Journal of Medicine, vol. 328, no. 10, pp. 708-716, 1993.

[7] K. P. Pruessmann, "Encoding and reconstruction in parallel MRI," NMR in Biomedicine, vol. 19, no. 3, pp. 288299, May 2006.

[8] M. Lustig, D. Donoho, and J. M. Pauly, "Sparse MRI: The application of compressed sensing for rapid MR imaging," Magnetic Resonance in Medicine, vol. 58, no. 6, pp. 1182-1195, Dec. 2007.

[9] G. Puy, J. P. Marques, R. Gruetter, J. Thiran, D. V. D. Ville, P. Vandergheynst, and Y. Wiaux, "Spread spectrum magnetic resonance imaging," IEEE Transactions on Medical Imaging, vol. 31, no. 3, pp. 586-598, Mar. 2012.

[10] D. Idiyatullin, C. Corum, J.-Y. Park, and M. Garwood, "Fast and quiet MRI using a swept radiofrequency," Journal of Magnetic Resonance, vol. 181, no. 2, pp. 342349, 2006.

[11] J. Tropp, M. Wakin, M. Duarte, D. Baron, and R. Baraniuk, "Random filters for compressive sampling and reconstruction," in IEEE International Conference on Acoustics, Speech and Signal Processing (ICASSP 2006), vol. 3, Toulouse, France, May 14-19 2006.

[12] J. C. Sprott, Chaos and time-series analysis. Oxford University Press Oxford, 2003.

[13] D. Idiyatullin, S. Suddarth, C. A. Corum, G. Adriany, and M. Garwood, "Continuous SWIFT," Journal of Magnetic Resonance, vol. 220, pp. 26-31, July 2012.

[14] M. Weiger, F. Hennel, and K. P. Pruessmann, "Sweep MRI with algebraic reconstruction," Magnetic Resonance in Medicine, vol. 64, no. 6, pp. 1685-1695, Dec. 2010.

[15] D. V. Phong, N. Linh-Trung, T. D. Tan, H. V. Le, and M. N. Do, "Fast image acquisition in magnetic resonance imaging by chaotic compressed sensing," in Proc. 8th IEEE International Symposium on Biomedical Imaging (ISBI 2011), Chicago, IL, USA, Mar. 30-Apr. 2 2011, pp. 85-88.

[16] T. Minh-Chinh, T. Tran-Duc, N. Linh-Trung, M. Luong, and M. N. Do, "Enhanced SWIFT acquisition with chaotic compressed sensing by designing the measurement matrix with hyperbolic-secant signals," in Prof. 34th Annual International Conference of the IEEE Engineering in
Medicine and Biology Society (EMBC 2012). San Diego, USA: IEEE, 2012, pp. 380-383.

[17] A. Bilgin, Y. Kim, H. G. Lalgudi, T. P. Trouard, and M. I. Altbach, "Parallel magnetic resonance imaging using compressed sensing," in Proc. SPIE 7073, Applications of Digital Image Processing XXXI, A. G. Tescher, Ed., Sep. 15 2008.

[18] T. D. Tan, D. V. Phong, T. M. Chinh, and N. Linh-Trung, "Accelerated parallel magnetic resonance imaging with multi-channel chaotic compressed sensing," in Proc. 3rd International Conference on Advanced Technologies for Communications (ATC 2010), Ho Chi Minh city, Vietnam, Oct. 20-22 2010, pp. 146-151.

[19] K. Pruessmann, M. Weiger, M. Scheidegger, and Boesiger, "SENSE: Sensitivity encoding for fast MRI," Magnetic Resonance in Medicine, vol. 42, no. 5, pp. 952-962, Nov. 1999.

[20] M. F. Duarte and Y. C. Eldar, "Structured compressed sensing: From theory to applications," IEEE Transactions on Signal Processing, vol. 59, no. 9, pp. 4053-4085, Sep. 2011.

[21] R. Calderbank, S. Howard, and S. Jafarpour, "Construction of a large class of deterministic sensing matrices that satisfy a statistical isometry property," IEEE Journal of Selected Topics in Signal Processing, vol. 4, no. 2, pp. 358-374, Apr. 2010.

[22] L. Yu, J. P. Barbot, G. Zheng, and H. Sun, “Compressive sensing with chaotic sequence," IEEE Signal Processing Letters, vol. 17, no. 8, pp. 731-734, Aug. 2010.

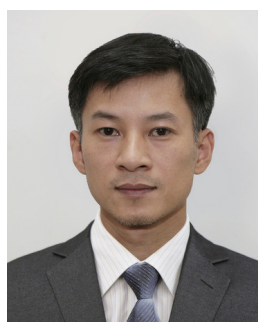

Nguyen Linh-Trung received both the B.Eng. and Ph.D. degrees in Electrical Engineering from Queensland University of Technology, Brisbane, Australia. From 2003 to 2005, he had been a postdoctoral research fellow at the French National Space Agency (CNES). He joined the University of Engineering and Technology within Vietnam National University, Hanoi, in 2006 and is currently an associate professor at its Faculty of Electronics and Telecommunications. He has held visiting positions at Telecom ParisTech, Vanderbilt University, Ecole Supérieure d'Electricité (Supelec) and the Université Paris 13 Sorbonne Paris Cité. His research focuses on methods and algorithms for data dimensionality reduction, with applications to biomedical engineering and wireless communications. The methods of interest include timefrequency analysis, blind source separation, compressed sensing, and network coding. He was co-chair of the technical program committee of the annual International Conference on Advanced Technologies for Communications (ATC) in 2011 and 2012. He is a senior member of the IEEE.

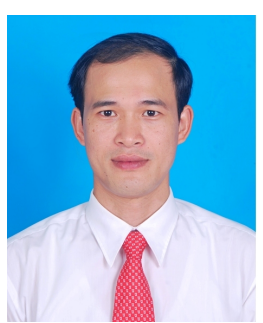

Truong Minh Chinh is a lecturer at the College of Education, Hue University. He obtained his B.Eng from Hanoi University of Science and Technology in 2003 and M.Sc. from the University of Engineering and Technology (UET), Vietnam National University Hanoi (VNU) in 2010. He is currently pursuing the PhD studies at the UET on compressed sensing. 


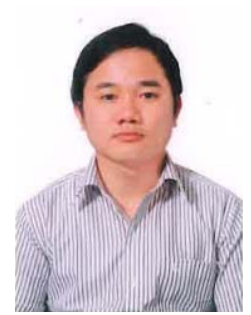

Tran Duc Tan was born in 1980. He received his B.Sc., M.Sc., and Ph.D. degrees respectively in 2002, 2005, and 2010 at the University of Engineering and Technology (UET), Vietnam National University Hanoi, Vietnam (VNU), where he has been a lecturer since 2006. He was the recipient of the Vietnam National University, Hanoi, Vietnam Young Scientific Award in 2008. He is currently an Associate Professor with the Faculty of Electronics and Telecommunications, University of Engineering and Technology, Vietnam National University, Hanoi, Vietnam. He is author and coauthor of 30 papers on MEMS based sensors and their application. His present research interest is in DSP applications.

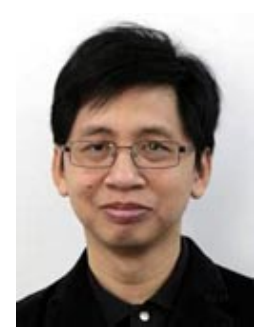

Ha Vu Le is currently with the Signals and Systems Laboratory, University of Engineering and Technology, Vietnam National University, Hanoi. He received the B.E. degree in Computer Science from Hanoi University of Technology in 1993. He was employed at the Institute of Information Technology, Vietnam, from 1993 to 1997, as a researcher, working to develop software tools and applications in the areas of computer graphics and geographical information systems. He received the M.S. degree from the California State Polytechnic University, Pomona, in 2000, and the Ph.D. degree from the University of Louisiana at Lafayette in 2003, both in Computer Science. His research interests include computer vision, image processing, biomedical signal processing, and automatic image/video annotation.

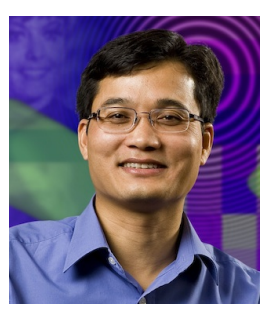

Minh N. Do was born in Thanh Hoa, Vietnam in 1974. He received the B.Eng. degree in Computer Engineering from the University of Canberra, Australia in 1997, and the Dr.Sci. degree in Communication Systems from the Swiss Federal Institute of Technology Lausanne (EPFL), Switzerland in 2001.

Since 2002, he has been on the faculty at the University of Illinois at Urbana-Champaign, where he is currently an Associate Professor in the Department of Electrical and Computer Engineering, and holds joint appointments with the Coordinated Science Laboratory, the Beckman Institute for Advanced Science and Technology, and the Department of Bioengineering. His research interests include image and multi-dimensional signal processing, computational imaging, wavelets and multiscale geometric analysis, and visual information representation.

He received a Silver Medal from the 32nd International Mathematical Olympiad in 1991, a University Medal from the University of Canberra in 1997, a Doctorate Award from the Swiss Federal Institute of Technology Lausanne in 2001, and a CAREER Award from the National Science Foundation in 2003. He co-authored on two papers with Arthur L. da Cunha and Ha T. Nguyen that received Best Student Paper Awards at the IEEE International Conference on Acoustics, Speech, and Signal Processing (ICASSP) in 2005, a paper with Yue Lu that received a Most Innovative Paper Award at the IEEE International Conference on Image Processing (ICIP) in 2006, and a paper with Yue Lu that received a Student Paper Award at ICIP in 2007. He received a Young Author Best Paper Award from the IEEE Signal Processing Society in 2008 for a paper co-authored with Martin Vetterli.

He was named a Beckman Fellow at the Center for Advanced Study, UIUC in 2006, and received a Xerox Award for Faculty Research, College of Engineering, UIUC, in 2007. He was a member of the IEEE Signal Processing Theory and Methods and Image, Video, and Multidimensional Signal Processing Technical Committees, and an Associate Editor of the IEEE Transactions on Image Processing. 